\title{
Community paramedicine is growing in impact and potential
}

\author{
Michael J. Nolan MA, Katherine E. Nolan BHSc, Samir K. Sinha MD DPhil
}

— Cite as: CMAJ 2018 May 28;190:E636-7. doi: 10.1503/cmaj.180642

See related article at www.cmaj.ca/lookup/doi/10.1503/cmaj.170740

I ncreasingly, paramedics are being recognized as uniquely positioned to support sustainable and high-quality health care delivery to Canada's aging population. In some Canadian jurisdictions, unnecessary 911 calls and emergency department visits have been rising by as much as $8 \%$ annually. ${ }^{1}$ Because paramedics initiate care for people in their own homes and communities, these health care professionals are well placed to recognize the unmet needs of the community-dwelling individuals they serve and to act proactively to support efforts to stem unnecessary use of emergency medical services.

The umbrella term, "community paramedicine," describes a growing field that began as a grassroots movement among paramedics who recognized a need and had the flexibility to create new services that emphasized a more proactive and preventive approach to care that utilizes paramedics in expanded roles. ${ }^{2}$ For example, across Canada and elsewhere, paramedics are increasingly conducting scheduled preventive home visits to improve chronic disease management in rural and remote regions. ${ }^{3}$ In other models, paramedics are using their unscheduled emergency encounters as an opportunity to refer their patients with unmet needs to home and community care services to prevent future unnecessary calls to 911 services and transports to the emergency department. ${ }^{4}$ These community paramedicine programs have been shown to improve care for community-dwelling individuals and reduce the unnecessary use of costly ambulance and hospital-based services. ${ }^{3,4}$

Research around this novel and rapidly evolving area of practice, however, remains limited. Two recent systematic reviews identified only 11 studies that evaluated community paramedicine and concluded that, although these programs appeared promising, further research about their effectiveness was needed. ${ }^{1,5}$ In linked research, Agarwal and colleagues describe a cluster randomized trial of a preventive wellness clinic-based model of community paramedicine within social-housing buildings. ${ }^{6}$ The trial's Community Paramedicine at Clinic (CP@clinic) chronic disease risk assessment and management program is a unique example of a partnership between community paramedics, primary care and providers of social housing to address a variety of unmet patient needs among a vulnerable population that

\section{KEY POINTS}

- Increasingly, paramedics are being recognized as uniquely positioned to deliver sustainable and high-quality health care in the community.

- Community paramedicine began as a grassroots movement among paramedics who recognized a need for new services that emphasized a more proactive and preventive approach to care by using paramedics in expanded roles.

- The findings of several studies suggest that community paramedicine programs can be effective in improving health outcomes for some populations, can reduce use of emergency services and may lower costs to the health care system.

are frequent users of 911 services and characterized as having higher mortality rates and poorer health-related quality of life and health because of higher rates of chronic disease among this population. This trial contributes further empiric evidence about the effect and potential for widespread adoption of community paramedicine programs.

Community paramedics have developed such pragmatic and collaborative approaches to care because they are a ubiquitous, highly skilled and mobile segment of the workforce who are interested in bridging gaps between service providers and stakeholders. These efforts are proving to be effective at both improving the quality and experience of patient care, and reducing overall costs of health care. ${ }^{7}$

The study by Agarwal and colleagues shows that patients with chronic conditions can benefit from improved access to health care resources and services in a familiar setting through the establishment of trusting relationships with local providers of paramedic services. ${ }^{6}$ The trial further highlights the potential value of deploying a low-cost community paramedicine intervention in a high-risk social-housing setting, because it showed a significant difference in the number of ambulance calls between participants who received the intervention (i.e., attending the CP@ clinic) and controls.

Although improvements in health-related quality of life and function reported at the patient level are important, it is the 
potential of community paramedicine programs to reduce future ambulance calls and resulting visits to the emergency department and, as a result, wait times for both emergency department and routine paramedic services, that is motivating jurisdictions across Canada to continue to invest in the development of community paramedicine models. Indeed, in rural Nova Scotia, a community paramedicine program developed to address a shortage of available primary care services reduced annual trips to emergency departments by $40 \%$ and decreased overall annual expenses for health care from $\$ 2380$ to $\$ 1375$ per person. ${ }^{7}$ In rural Ontario, a community paramedicine program targeted around medically complex high users reduced 911 activation by $24 \%$, emergency department visits by $20 \%$ and admissions to hospital after emergency department visits by $55 \%$ one year after implementation. ${ }^{8}$ Despite consistency of findings, the before-and-after design of these and most current studies will always limit the interpretation of their results because "regression to the mean" cannot be ruled out. Therefore, the findings from Agarwal and colleagues' trial ${ }^{6}$ help to strengthen the evidence base that supports the effectiveness of initiatives in community paramedicine for both patient and system outcomes.

As Canada's population ages and the complexity of patients' needs continues to increase, heath care systems will need to adapt resource allocation to ensure improved patient care and system efficiency. The promise and potential of paramedicine practice to evolve beyond traditional emergency response is being realized across Canada and beyond. As governments continue to invest in the expansion of these services, service standards are being established, ${ }^{9}$ and standardized training of paramedics to provide community paramedicine programs is becoming commonplace. To support these efforts, more robust research is required to define an evidence-based framework on which to base new and existing programs that will further advance the delivery of health care and the overall sustainability of our health care systems.

\section{References}

1. Bigham BL, Kennedy S, Morrison L. Expanding paramedic scope of practice in the Community: a systematic review of the literature. Prehosp Emerg Care 2013; 17:361-72.

2. lezzoni LI, Dorner SC, Ajayi T. Community paramedicine - addressing questions as programs expand. N Engl J Med 2016;374:1107-9.

3. Ruest M, Stitchman A, Day C. Evaluating the impact on 911 calls by an in-home programme with a multidisciplinary team. Int Paramedic Practice 2012;2:125-32.

4. Verma AA, Klich J, Thurston A, et al. Paramedic-initiated home care referrals and use of home care and emergency medical services Prehosp Emerg Care 2018;22:379-84.

5. Condino AE. The impact of community paramedicine programs on the health of rural communities in the United States and Canada: a systematic review. Ann Emerg Med 2016;68:S71.

6. Agarwal G, Angeles R, Pirrie M, et al. Evaluation of a community paramedicine health promotion and lifestyle risk assessment program for older adults who live in social housing buildings: a cluster randomized trial. CMAJ 2018;190:E638-47.

7. White R, Wingrove G. Principles for community paramedicine programs [policy brief]. Washington (DC): National Rural Health Association; 2012. Available: www.ruralhealthweb.org/getattachment/Advocate/Policy-Documents/Principlesfor CommunityParamedicineSept-2012.pdf.aspx?lang=en-US (accessed 2018 May 9).

8. Ruest MR, Ashton CW, Millar J. Community Health Evaluations Completed Using Paramedic Service (CHECUPS): design and implementation of a new community-based health program. J Health Hum Serv Adm 2017;40:186-218.

9. Community paramedicine framework for planning, implementation and evaluation Toronto: Ministry of Health and Long-Term Care (Home and Community Care Branch); 2017. Available: www.renfrewparamedics.ca/wp-content/uploads /2017/07/CP-Framework-2017_07_18.pdf (accessed 2018 May 10).

\section{Competing interests: None declared.}

This article was solicited and has not been peer reviewed.

Affiliations: County of Renfrew (M. Nolan, K. Nolan), Renfrew, Ont.; Division of General Internal Medicine and Geriatrics, Department of Medicine (Sinha), Sinai Health System and University Health Network; Department of Medicine (Sinha); Institute of Health Policy, Management and Evaluation (Sinha), University of Toronto, Toronto, Ont.

Contributors: Katherine Nolan performed the literature review. All of the authors drafted the manuscript, revised it critically for important intellectual content, gave final approval of the version to be published and agreed to be accountable for all aspects of the work.

Correspondence to: Michael Nolan, MNolan@countyofrenfrew.on.ca 